Published in final edited form as:

J Res Pers. 2008 December ; 42(6): 1448-1455. doi:10.1016/j.jrp.2008.06.006.

\title{
Personality Predictors of the Time Course for Lung Cancer Onset
}

\author{
Adam A Augustine, \\ Washington University in St. Louis \\ Randy J. Larsen, \\ Washington University in St. Louis
}

Mark S. Walker, and

Accelerated Community Oncology Research Network, Inc.

Edwin B. Fisher

University of North Carolina, Chapel Hill

\begin{abstract}
Numerous findings suggest that personality is linked to the incidence and experience of negative health outcomes. More specifically, trait negative affect is negatively related to a number of health outcomes. The current study expands our understanding of the link between personality and disease by examining the time course for lung cancer onset. In a sample of patients who had recently undergone surgical resection for lung cancer, a variety of negative affect-related personality variables were assessed to determine their relationship with age at surgery. After controlling for smoking behavior, it was found that trait negative affect was associated with time course for lung cancer onset, such that those with higher (vs. lower) levels of trait negative affect manifested lung cancer earlier in their lives. Thus, trait negative affect represents an independent risk factor among those prone to lung cancer (i.e. smokers).
\end{abstract}

\section{Keywords}

lung cancer; negative affect; personality; depression; anxiety

The idea that personality influences health lies at the heart of an increasing amount of research in both psychological and medical literatures. Indeed, numerous findings suggest that personality is linked to the incidence of negative health outcomes, such as cancer and chronic heart disease (Suls \& Bunde, 2005; Friedman \& Booth-Kewley, 1987). Personality may be related to disease through personality-specific health behaviors (health behavior models), stress appraisals and coping behavior (interactional stress moderation models), exposure to stressful events (transactional stress moderation models), or the presence of an underlying variable related to both personality and disease (constitutional predisposition models; Smith \& Gallo, 2001; Smith, Glazer, Ruiz, \& Gallo, 2004). No matter the particular model of personality and health one endorses, it is clear that personality and health are related. Additionally, personality

(C) 2008 Elsevier Inc. All rights reserved.

Correspondence concerning this article should be addressed to Adam A Augustine, Washington University in St. Louis, Department of Psychology, Campus Box 1125, One Brookings Drive, St. Louis, MO, 63130-4899. Electronic mail may be sent to aaaugust@artsci.wustl.edu.

Publisher's Disclaimer: This is a PDF file of an unedited manuscript that has been accepted for publication. As a service to our customers we are providing this early version of the manuscript. The manuscript will undergo copyediting, typesetting, and review of the resulting proof before it is published in its final citable form. Please note that during the production process errors may be discovered which could affect the content, and all legal disclaimers that apply to the journal pertain. 
risk factors may function either independently (Grossarth-Maticek \& Eysenck, 1995) or in a synergistic/additive manner with other risk factors (Marusic \& Eysenck, 2001).

The current study attempts to expand our understanding of the link between personality and disease by examining a relatively novel outcome variable, age at time of lung cancer surgery. In a sample of patients who had recently undergone surgical resection for stage I or II nonsmall-cell lung cancer, a variety of personality variables were assessed to determine their relationship with age at surgery. Broad links between personality and a wide array of disease outcomes suggests that personality may play a role in how soon one is surgically treated for lung cancer, or the time course for lung cancer onset.

Neuroticism is a consistent predictor of health outcomes, with those higher (vs. lower) in neuroticism experiencing shorter life spans and a greater incidence of serious illness (Suls \& Bunde, 2005). Neurotics (vs. emotionally stable) are more likely to engage in lifestyle activities that lead to cancer, including smoking (van Loon, Tijhuis, Surtees, \& Ormel, 2001; Terracciano \& Costa, 2004; Munafo, Zetteler, \& Clark, 2007). In line with this finding, those who are diagnosed with cancer are likely to be higher in neuroticism relative to those without cancer (Amelang, 1997). Trait anxiety is similar to neuroticism, and findings relating anxiety to disease follow the same pattern as those relating neuroticism to disease. Individuals who are higher (vs. lower) in trait anxiety show a greater incidence of serious disease. In a prospective study, Weihs, Enright, Simmons, and Reiss (2000) found that low anxiety predicted longer survival after breast cancer. Trait anxious individuals are also more likely to experience asthma, ulcers, arthritis, and headaches (Friedman \& Booth-Kewley, 1987). Additionally, trait anxiety positively predicts the incidence of chronic heart disease even when controlling for other physical and behavioral causes of the disease (Suls \& Bunde, 2005). Finally, some types of intervention for anxiety (or depression) have been found to increase cancer survival (Ross, Boesen, Dalton, \& Johansen, 2002).

Anger (and its related trait level behaviors of hostility and aggression) has also been linked with the experience of serious illness, with those higher (vs. lower) in trait anger/aggression/ hostility experiencing greater incidence of serious disease. In an outpatient sample, Glover, Dibble, Dodd, and Miaskowski (1995) found that anger was related to the experience of more cancer-related pain. Additionally, trait anger/hostility is linked to greater incidence of chronic heart disease, asthma, and arthritis (Friedman \& Booth-Kewley, 1987). Finally, Smith et al. (2004) found that, of all the components of Type A personality, it was the anger/hostility component that was the strongest predictor of chronic heart disease.

Depression, whether clinical or not, is associated with asthma, ulcers, arthritis, and headaches, with those higher in depression experiencing more instances of these illnesses (Friedman \& Booth-Kewley, 1987). Depression positively predicts: death from breast cancer (Goodwin, Zhang, \& Ostir, 2004), the risk of cancer (Jacobs \& Bovasso, 2000), and the incidence of chronic heart disease even when other physical and behavioral causes are controlled (Suls \& Bunde, 2005). Those who consistently use a depressive style of coping are also more prone to cancer. Individuals that cope by ruminating, arguing with fate, pitying themselves, withdrawing, and taking out their feelings have a shorter period of survival following lung cancer diagnosis (Faller, Bulzebruck, Drings, \& Lang, 1999; Faller \& Schmidt, 2004). In addition, a repressive coping pattern is associated with an increased incidence of breast cancer (McKenna, Zevon, Corn, \& Rounds, 1999) and a passive coping pattern is associated with shorter survival of breast cancer (Gerits, 2000).

All of the personality variables described above have been linked to the incidence and experience of serious illness. These personality variables also share a common theme, negative affect. Neuroticism, anger/hostility, anxiety, depression, and less adaptive coping patterns are 
all indicative of individuals who experience frequent, intense, and long lasting negative affect. Suls and Bunde (2005) propose that it is not the experience of specific negative affects (i.e. anger, sadness, etc.) but rather a predisposition to the experience of global negative affect that is of the most importance in the affect-disease relationship. The links between these negative affect laden traits and disease outcomes suggest that those who experience more negative affect may reach a point of surgical cancer intervention sooner than those who experience less negative affect. However, some data indicates that this may not be the case.

A number of studies and literature reviews have concluded that evidence for the link between trait negative affect and various cancer-related outcomes is inconclusive. First, in a large prospective study, Alamada, Zonderman, Shekelle, Dyer, Daviglus, Costa, and Stamler (1991) found that, although neuroticism was associated with cancer risk factors (i.e., smoking) and non-cardiovascular, non-cancer disease risk, it was not independently associated with risk for cancer development. Second, several reviews of the literature on psychosocial predictors of breast cancer have concluded that significant results are inconsistent, even for prospective studies (Bleiker \& van der Ploeg, 1999; Butow, Hiller, Price, Thackway, Kricker, \& Tennant, 2000). Third, although Faller and Schmidt (2004) did find that depressive coping was associated with lower survival from lung cancer, they did not observe links between depression and cancer survival. Fourth, in a meta-analysis, Petticrew, Bell, and Hunter (2002) concluded that coping style was not associated with cancer survival. Finally, in a review of prospective studies on psychosocial predictors of cancer development, Garssen (2004) concluded that, although depression and lack of social support seem to predict faster development of cancer, no evidence for links between cancer development and psychosocial factors is particularly convincing.

These findings suggest that the relationship between personality and disease (particularly cancer) may be more complex than was previously thought. However, as is often pointed out, methodology seems to play a key role in whether or not these relationships are observed. First, the population examined may impact observed relationships. A majority of these studies examined predictors of the risk for cancer and, thus, examine all individuals in the relevant analyses. However, the personality-disease effects may only appear in specific populations. In line with this, Linkins and Comstock (1990) found (using a prospective design) that anhedonic or depressive trait affect was weakly associated with the development of cancer when examining all individuals, but this relationship was strong among smokers. Second, the type of measurement tools used may also play a role. In several reviews (i.e., Garssen, 2004) it has been noted that prospective studies do not always yield the significant results that are common in retrospective studies. While this does seem to be the case, most prospective studies also share a common feature; they utilize clinical or non-traditional measures rather than traditional psychological measures of personality. Using a retrospective design, the current study examines the negative affect - cancer link using psychologically based measures of personality in a population of individuals recently treated for lung cancer.

\section{The Current Study}

The current study attempts to address the relationship between several negative affect related personality traits and the age at which participants undergo surgical intervention for lung cancer. The primary dependent measure in this study (age at which participants underwent surgery) could be interpreted as either representing how long patients delayed before seeking treatment or how quickly patients show symptoms of lung cancer. Regarding the former, one study has found a relationship between personality and how long individuals wait before seeking medical treatment. In a retrospective study of rectal cancer patients, Ristvedt and Trinkhaus (2005) found that most individuals sought treatment immediately after symptoms appeared. Approximately $2 / 3$ of the variance in how long individuals waited to seek treatment 
was accounted for by ways in which individuals interpreted their symptoms (i.e. it's just foodpoisoning/stress/etc.). However, anxiety did predict delay in seeking treatment such that low (vs. high) anxious individuals waited longer to see a doctor.

While interpretation of the measure used in our study (age at which participants underwent surgery) as representing delay in seeking treatment may seem appropriate, several aspects of both the patient sample and the design of the study indicate that interpreting the measure as representing the time course for illness onset is more appropriate. First, the patient population is relatively homogenous in terms of the progression of their lung cancer; all underwent surgical resection for stage I or II non-small-cell lung cancer. By capturing the sample at a relatively similar stage of progression, time of first disclosure is somewhat irrelevant. Second, by controlling for behavioral risk factors (i.e. how long patients smoked, how much patients smoked) the relationship between negative affect laden personality traits and age at time of surgery should represent the unique effect that personality has on the speed of illness onset above and beyond these potent risk factors. Third, it has been shown that, even in countries with less developed health care systems, patients seek treatment for lung cancer symptoms very quickly after presentation (about 30 days) and that treatment begins within a few weeks of the initial consultation (Ozlu, Bulbul, Oztuna, \& Can, 2004). Thus, given the homogeneity of the sample, the risk-factor covariates included, and the short time frame that is typical between development of symptoms and medical intervention, the dependent variable in this study (age at time of surgery) is most representative of the time course for lung cancer onset. To further examine this issue, analyses will be conducted to determine the age differences for those high and low in those traits that significantly predict age at time of surgery. As lung cancer is already in an advanced stage once initial symptoms are visible, rapid progression or death usually results quickly (in a few years) after initial symptom presentation. Thus, large age differences (three to five years) between those high and low in the predictive traits would indicate, not an effect on treatment delay, but an effect on progression speed.

It is hypothesized that the presence of high (vs. low) trait negative affect will be related to age at time of surgery, such that those higher in trait negative affect receive lung cancer surgery at a younger age (earlier onset of illness). This study has three key strengths that will allow for the investigation of this hypothesis. First, the sample consists of individuals who had recently undergone surgical intervention for lung cancer. Second, important demographic and smoking behavior variables are controlled. Finally, a wide range of negative affect related personality variables are assessed using traditional personality assessment tools.

\section{Method}

Participants

Data presented in this study were collected as part of a larger longitudinal study examining individuals undergoing treatment at The Alvin J. Siteman Cancer Center at Barnes- Jewish Hospital and the Washington University School of Medicine. All participants were being treated for lung cancer and had recently undergone surgical resection for stage I or II nonsmall-cell lung cancer. A total of 203 (age $M=59.56, S D=10.00$, range $=33-85$ years old) participants were recruited for this study (participant characteristics can be viewed in Table $1)$.

\section{Materials}

Demographic and smoking related variables-A number of items assessed age, level of education, race, marital status, income level, and employment status. In addition, several items assessed smoking related demographic variables, such as age at time or surgery, age 
when participant began smoking, and amount participant smoked per day prior to surgery (see Table 1).

Personality-Personality was assessed using the Behavioral Activation/Behavioral Inhibition Scale (BAS/BIS: Carver \& White, 1994) and the Eysenck Personality Questionnaire (EPQ: Eysenck \& Eysenck, 1975). Reponses on the BAS/BIS scales are made on a 4-point, strongly agree - strongly disagree, Likert-type scale. The BAS/BIS scale yields four subscales: BAS Fun $(M=9.85, S D=1.88$, full BAS $\alpha=.80)$, BAS Reward Responsiveness $(M=9.83$, $S D=2.45)$, BAS Drive $(M=9.77, S D=2.21)$, and BIS $(M=15.97, S D=3.17, \alpha=.76)$.

Response on the EPQ are made on a 5-point, very inaccurate - very accurate, Likert-type scale. The EPQ yields four subscales: extraversion $(M=7.43, S D=3.44)$, neuroticism $(M=4.36$, $S D=3.60)$, psychoticism $(M=1.71, S D=1.58)$, and a lie $(M=5.77, S D=2.81)$ scale. Prior use of the EPQ has yielded high internal consistency (see Miles \& Hempel, 2004).

Anger/Aggression-Trait levels of anger and aggression were assessed using the BussPerry aggression questionnaire (BP aggression: Buss \& Perry, 1992). Responses on the BP aggression scale are made on a 7-point, extremely uncharacteristic of me - extremely characteristic of me, Likert-type scale. The BP aggression scale yields five subscales: anger $(M=14.39, S D=5.09)$, hostility $(M=16.87, S D=6.90)$, physical aggression $(M=17.57$, $S D=6.55)$, verbal aggression $(M=12.15, S D=4.14)$, and total aggression $(M=61.21, S D=$ 19.02; full scale $\alpha=.92$ ).

Anxiety-Trait anxiety was assessed using the State-Trait Anxiety Inventory (STAI: Spielberger, Gorsuch, Lushene, Vagg, \& Jacobs, 1983). Responses on the STAI are made on a 4- point, not at all - very much so, Likert-type scale $(M=40.21, S D=10.15, \alpha=.95)$.

Depression-Depression was assessed using an orally presented version of the Beck Depression Inventory (BDI: Beck \& Steer, 1984; $M=10.57, S D=7.82, \alpha=.80$ ).

Coping-Coping behavior was assessed using the COPE (Carver, Scheier, \& Weintraub, 1989). Reponses on the COPE are made on a 4-point, I haven't done this at all - I have done this a lot, Likert-type scale. Although there are a number of ways in which the COPE can be scored, for the purposes of this study, we chose to score the COPE using a two-subscale approach that yields scores for the use of adaptive $(M=2.63, S D=.62, \alpha=.84)$ and less adaptive coping strategies $(M=1.73, S D=.49, \alpha=.88)$.

\section{Procedure}

Eligible participants were identified by a physician's assistant working in the surgical ward and through the review of surgery schedules and records. Participants were recruited either during their inpatient stay (following surgery) or during the week following their release. Participants were contacted and the purpose of the study was described. After obtaining consent, a research assistant administered all questionnaires either in written or oral form.

\section{Results \\ Primary Analyses}

The primary purpose of this study is to determine which psychological variables predict the speed at which lung cancer becomes severe and requires surgery. As such, the dependent variable throughout these analyses is the age of the participant at the time of their surgery. To determine which psychological variables have predictive validity independent of smoking behavior, two variables are controlled throughout the following analyses: age participant started smoking and amount participant smoked per day prior to surgery. Age participant started 
smoking was not significantly related to age at time of surgery $(r=.11, p=.12)$ however, controlling for this variable will remove the effect of number of years smoked. Amount the participant smoked per day prior to surgery was significantly related to age at time or surgery $(r=-.17, p<.05)$ and is also controlled in the following analyses. Partial correlations between age at time of surgery and the psychological variables measure in this study (controlling for age participant started smoking and amount participant smoked prior to surgery) are presented in Table 2.

Results of the partial correlation analyses indicate that men, Caucasians, and the employed are older at the time of their surgery (later onset of illness) while women, minorities, and the unemployed are younger at the time of their surgery (earlier onset of illness). Neuroticism, BIS, anger, hostility, verbal aggression, total aggression, anxiety, and depression were all negatively related to age at time of surgery, such that those higher (vs. lower) in these constructs showed an earlier onset of illness (younger at surgery). Finally, both the use of adaptive and less adaptive coping strategies were negatively related to age at time of surgery, such that those who typically engage in more coping showed an earlier onset of illness (younger at surgery).

In an attempt to examine the role of delay in seeking treatment, we determined the size (in years) of these effects by predicting the age at surgery at one standard deviation above and below the mean for each of the significant personality predictors (controlling smoking behavior). Results indicate a mean difference (for all significant personality variables) of 4.00 years at one standard deviation above versus below the mean. Neuroticism (4.33), BIS (3.12), anger (3.26), hostility (4.13), verbal aggression (3.49), total aggression (3.69), anxiety (2.9), depression (7.04), less adaptive coping (5.11), and adaptive coping (3.04) all yielded age differences greater than 2.9 years.

\section{Supplementary Analyses}

As gender differences exist in several of the examined personality variables (particularly anger), a second set of analyses was conducted to examine the role of gender in the observed links between personality and age of lung cancer onset. Partial correlations controlling gender, as well as the two previously mentioned smoking behavior covariates (age began smoking and amount smoked per day), can be viewed in Table 2. The pattern of significant predictors was generally unchanged. However, the effect for BIS became non-significant and the effect for physical aggression became significant when gender was included as a covariate.

To examine the possibility that the significant predictors interact with gender to predict age of lung cancer onset, hierarchical multiple regression analyses were conducted (smoking covariates were entered on step 1, gender and the relevant personality variable on step 2 , and the interaction term on step 3$)$. Results indicate a significant BIS $x$ gender interaction $(\beta=.94$, $t(199)=2.44, p<.05)$ and a simple slopes follow-up test indicates that while men show the effect $(\beta=-.40, t(199)=2.15, p<.05)$, women do not show the effect $(\beta=-.16, t(199)=.85$, $n s)$. No other interaction effects where significant $(\beta s=-.41-.28, n s)^{1}$.

It is also possible that coping behavior could mediate the effects of negative affect on age of lung cancer onset. To examine this, mediation models (controlling smoking behavior and gender) were examined for neuroticism, total trait anger score, depression, and anxiety. Only the effect for neuroticism was reduced when coping behavior (less adaptive coping, but not adaptive coping reduced the effect) was entered into the regression equation. A test of the

\footnotetext{
${ }^{1}$ In addition to gender, it is possible that negative affectivity would interact with smoking behavior to predict age of onset. Hierarchical multiple regression analyses indicate that neither age participant started smoking $(\beta=-.82-.44, n s)$, nor cigarettes smoked per day $(\beta=$ $-.15-.33, n s)$ interacted with any of the significant personality predictors to predict age of onset.
} 
mediation path indicates that coping behavior does not significantly mediate the relationship between neuroticism and age of lung cancer onset (Sobel's $t=.01, n s$ ).

\section{Discussion}

In the current study, negative affect laden personality variables were assessed in a sample of lung cancer patients who had recently undergone surgical resection for stage I or II non-smallcell lung cancer. The purpose of this study was to determine the relationship between personality and the patients' age at the time of surgery. Due to the nature of the patient sample, our ability to control for smoking history and other risk factors, the typically brief delay (1-2 months) between presentation of symptoms and beginning of treatment, and the relatively large age at surgery differences observed for differing levels of trait negative affect, age at time of surgery is thought to represent the time course for lung cancer onset. Partial correlations indicate that (controlling for how long the participant smoked and how much the participant smoked each day) men, Caucasians, and the employed are older at the time of their surgery (later illness onset) while women, minorities, and the unemployed are younger at the time of their surgery (earlier illness onset). Neuroticism, BIS, anger, hostility, verbal aggression, total aggression, anxiety, and depression were negatively related to age at time of surgery such that those higher (vs. lower) in these constructs showed an earlier onset of illness (younger at surgery). Finally, coping behavior (both adaptive and less adaptive) was negatively related to age at time of surgery, such that those who engage in more coping showed an earlier onset of illness (younger at surgery). This general pattern of effects emerges even when controlling for gender (although BIS does interact with gender such that men show the effect and women do not).

Although a review of the literature may yield inconsistent patterns of findings for the personality-cancer link, this study examined a specific population with traditional psychological measures and did find the oft hypothesized (and criticized) link between negative affectivity and cancer. While a number of studies find no link between negative affect and cancer in the general population, Linkins and Comstock (1990) found that, while negative affect is weakly associated with the development of cancer in the general populace, this relationship is strong among smokers (and our sample consisted of smokers).

Recent evidence from three genome wide searches for genetic markers of disease reveal that variations in genes regulating nicotinic acetylcholine receptors either directly influence or mediate the effects of smoking on lung cancer development; these receptors are active in a region of the brain associated with depression (for a review of these studies, see Chanock \& Hunter, 2008). In other words, the development of lung cancer among smokers is related to depression at a genetic/neurological level. Thus, consistent with prior research, this study indicates that, among those who develop lung cancer, individuals with higher (vs. lower) levels of negative affect manifest lung cancer earlier in their lives. High (vs. low) levels of neuroticism, BIS, depression, anxiety, and hostility/anger/aggression are related to an earlier onset of lung cancer among those at risk for lung cancer. While these negative affect variables may not predict who is eventually diagnosed with lung cancer, they are clearly involved in the development of lung cancer among those at risk for the disease.

It is possible that trait negative affect is related to the speed of cancer progression due to its impact on the immune system. The experience of negative affect leads to depletion of the immune system (Irwin, 2002). Those who experience frequent, intense, and long lasting negative affect may posses a consistently depleted immune response and thus, be less able to fight off the progression of lung cancer. In other words, those with heightened trait negative affect might not possess the immunological resources needed to resist the progression of a lung cancer infection. 
It is also possible that the relationships between age of onset and negative affect may be due to diagnosis reactivity, such that younger individuals "take the diagnosis harder." There are several lines of reasoning which suggest that this explanation is not relevant for our results. First, one could reasonably assume that a lung cancer diagnosis would create a ceiling effect for state negative affect; all individuals would immediately reach a very high level of negative affect upon learning of their illness. Still, younger individuals may react with more state negative affect when they are diagnosed. However, in this study, we utilized trait, not state measures of negative affectivity and personality traits are typically not susceptible to rapid alterations. Second, if a reactivity explanation were the case, one would not expect to see any relationships between coping and age of onset; a lung cancer diagnosis should not differentially impact the coping behavior of older vs. younger individuals. However, in this study relationships between coping behavior and age of onset were observed. Finally, the overwhelming majority of participants in this study were over 50 years of age (age $M=59.56$, $S D=10.00)$. It is unclear why a cancer diagnosis would differentially effect people that were 50,60 , or 70 at the time of their diagnosis. Additionally, at this point in the lifespan, personality is largely static. Thus, given ceiling effects, our examination of trait (not state) negative affect, the observed effects for coping, and the age of the sample, it is very unlikely that differential reactivity to the diagnosis is driving the observed effects.

The finding that more use of any coping strategy is associated with a faster illness progression may seem odd at first glance. However, it is important to bear in mind that individuals who experience more negative affect do engage in more coping. It should also be noted that the effect size for the use of less adaptive coping strategies is larger than the effect size for the use of adaptive coping strategies. This suggests that, although those higher in trait negative affect engage in all coping more frequently, the use of less adaptive coping strategies may be particularly damaging to one's health. Those who use less adaptive coping strategies likely experience higher levels of negative affect (as a consequence of maladaptive coping) and thus, exhibit a faster progression of lung cancer.

\section{Limitations and Future Directions}

The primary dependent measure in this study was the age at which patients underwent surgical intervention for lung cancer. This measure is interpreted as representing the time course for lung cancer onset. Given the roughly equivalent stage of lung cancer across patients, the smoking behavior covariates included in this study, and the typically short delays between the initial manifestation of symptoms and treatment (Ozlu, Bulbul, Oztuna, \& Can, 2004) this seems the most likely explanation for the effects observed. In addition, lung cancer is already at an advanced stage once initial symptoms are noticeable and a rapid progression (beyond the tumor sizes treated in this study) or death usually results within a few years of symptom appearance. Given that those high versus low in the relevant personality variables differed in age by at least three years (four years for neuroticism and seven for depression), the observed effects do not likely represent treatment delay; those individuals high in these traits would have been at far more advanced stages and, likely, would have been deceased. However, one still might argue that this measure (age at time of surgery) represents delay of disclosure. Even if this were the case, the findings would still be of some importance. The earlier one gains treatment for any cancer, the better that person's chances of survival. Given this alternate interpretation of the dependent measure, one would find that worriers (i.e. those possessing higher levels of trait negative affect) seek treatment sooner (similar to the findings for colon cancer patients; Ristvedt \& Trinkhaus, 2005). Thus, it would be important to encourage those low in negative affect to seek treatment for possible cancer symptoms more quickly. However, given the nature of the sample, the controls included, the low delays in seeking treatment for lung cancer, and the relatively large differences in age for those high versus low in these traits, this alternative explanation is not likely the case. 
The results of this study suggest several directions for future research regarding the personalitydisease link. First, some types of intervention for negative affectivity have been found to increase cancer survival (Ross, Boesen, Dalton, \& Johansen, 2002). As high negative affect is associated with a faster speed of lung cancer development among smokers, it is important to investigate the efficacy of negative affect treatment programs for those at risk for cancer (i.e., smokers). Even if those at risk do not display clinical levels of depression or anxiety, therapy or pharmacological treatments known to alleviate depressive or anhedonic conditions may help to slow the course of cancer. Second, when viewed in concert with the findings of Linkins and Comstock (1990), the findings of this study suggest that it is important to consider the sample in studies examining personality - cancer links. When examining the general populace, mean level differences in personality may not exist between those who never develop and those who do develop cancer. However, personality may represent a risk factor among certain individuals, such as smokers. Finally, if one is to make judgments regarding the effect of personality factors on various cancer outcomes, it is important to use psychological measures and not those designed for clinical uses (i.e., hospital depression scales). If it is personality, and not some momentary or clinically significant state that is to be investigated, then standard personality assessments should be used.

In sum, among those at risk for lung cancer (i.e., smokers) trait negative affect is associated with a faster speed of lung cancer onset. Indeed, the effect sizes for negative affect are generally as large as, and independent from, smoking behavior itself. Thus, trait negative affect is, in the least, as important (statistically speaking) as smoking behavior in the time course for lung cancer onset.

\section{References}

Alamada SJ, Zonderman AB, Shekelle RB, Dyer AR, Daviglus ML, Costa PT, Stamler J. Neuroticism and cynicism and risk of death in middle-aged men: The western electric study. Psychosomatic Medicine 1991;53:165-175. [PubMed: 2031070]

Amelang M. Using personality variables to predict cancer and heart disease. European Journal of Personality Special Issue: Personality and Chronic Disease 1997;11:319-342.

Beck AT, Steer RA. Internal consistencies of the original and revised Beck Depression Inventory. Journal of Clinical Psychology 1984;40:1365-1367. [PubMed: 6511949]

Bleiker EM, van der Ploeg HM. Psychosocial factors in the etiology of breast cancer: review of a popular link. Patient Education and Counseling 1999;37:201-214. [PubMed: 14528547]

Buss AH, Perry MP. The aggression questionnaire. Journal of Personality and Social Psychology 1992;63:452-459. [PubMed: 1403624]

Butow PN, Hiller JE, Price MA, Thackway SV, Kricker A, Tennant CC. Epidemiological evidence for a relationship between life events, coping style, and personality factors in the development of breast cancer. Journal of Psychosomatic Research 2000;49:169-181. [PubMed: 11110988]

Carver CS, Scheier MF, Weintraub JK. Assessing coping strategies: a theoretically based approach. Journal of Personality and Social Psychology 1989;56:267-283. [PubMed: 2926629]

Carver CS, White TL. Behavioral inhibition, behavioral activation, and affective responses to impending reward and punishment: The BIS/BAS scales. Journal of Personality and Social Psychology 1994;67:319-333.

Chanock SJ, Hunter DJ. When the smoke clears... Three studies identify an association between genetic variation at a location on chromosome 15 and risk of lung cancer. But they disagree on whether the link is direct or mediated through nicotine dependence. Nature 2008;452:537-538. [PubMed: 18385720]

Eysenck, HJ.; Eysenck, SBG. Manual of the Eysenck Personality Questionnaire. San Diego, CA: Educational and Industrial Testing Service; 1975.

Faller H, Bulzebruck H, Drings P, Lang H. Coping, distress, and survival among patients with lung cancer. Archive of General Psychiatry 1999;56:756-762. 
Faller H, Schmidt M. Prognostic value of depressive coping and depression in survival of lung cancer patients. Psycho-Oncology 2004;13:359-363. [PubMed: 15133776]

Friedman HS, Booth-Kewley SB. The "Disease-Prone Personality:" A meta-analytic view of the construct. American Psychologist 1987;42:539-555. [PubMed: 3619180]

Garssen B. Psychological factors and cancer development: Evidence after 30 years of research. Clinical Psychology Review 2004;24:315-338. [PubMed: 15245834]

Gerits P. Life events, coping, and breast cancer: state of the art. Biomedicine and Pharmacotherapy 2000;54:229-233.

Glover J, Dibble SL, Dodd MJ, Miaskowski C. Mood states of oncology outpatients: Does pain make a difference? Journal of Pain and Symptom Management 1995;10:120-128. [PubMed: 7730684]

Goodwin JS, Zhang DD, Ostir GV. Effects of depression on diagnosis, treatment, and survival of older women with breast cancer. Journal of the American Geriatrics Society 2004;52:106-111. [PubMed: 14687323]

Grossarth-Maticek R, Eysenck HJ. Self-regulation and mortality from cancer, coronary heart disease, and other causes: A prospective study. Personality and Individual Differences 1995;19:781-795.

Irwin M. Psychoneuroimmunology of depression: Clinical implications. Brain, Behavior, and Immunity 2002;16:1-16.

Jacobs JR, Bovasso GB. Early and chronic stress and their relation to breast cancer. Psychological Medicine 2000;30:669-678. [PubMed: 10883721]

Linkins RW, Comstock GW. Depressed mood and the development of cancer. American Journal of Epidemiology 1990;134:324-326.

Marusic A, Eysenck HJ. Synergistic interaction of smoking and neuroticism as a risk factor in ischaemic heart disease: case-control study. Personality and Individual Differences 2001;30:47-57.

Miles, J.; Hempel, S. The Eysenck personality scales: The Eysenck Personality Questionnaire-Revised (EPQ-R) and the Eysenck Personality Profiler (EPP). In: Hilsenroth, MJ.; Segal, DL., editors. Comprehensive Handbook of Psychological Assessment: Personality Assessment. Hoboken, NJ: John Wiley and Sons; p. 99-107.

Munafo MR, Zetteler JI, Clark TG. Personality and smoking status: A meta-analysis. Nicotine \& Tobacco Research 2007;9:405-413. [PubMed: 17365772]

Ozlu T, Bulbul Y, Oztuna F, Can G. Time course from first symptom to the treatment of lung cancer in the Eastern Black Sea region of Turkey. Medical Principles and Practice 2004;13:211-214. [PubMed: 15181326]

Petticrew M, Bell R, Hunter D. Influence of psychological coping on survival and recurrence in people with cancer: A systematic review. British Medical Journal 2002;325:1066-1076. [PubMed: 12424165]

Ristvedt SL, Trinkhaus KM. Psychological factors related to delay in consultation for cancer symptoms. Psycho-Oncology 2005;14:339-350. [PubMed: 15386764]

Ross L, Boesen EH, Dalton SO, Johansen C. Mind and cancer: Does psychosocial intervention improve survival and psychological well-being? European Journal of Cancer 2002;38:1447-1457. [PubMed: 12110489]

Smith, TW.; Gallo, LC. Personality traits as risk factors for physical illness. In: Baum, A.; Revenson, T.; Singer, J., editors. Handbook of Health Psychology. Hilsdale, NJ: Lawrence Erlbaum Associates; 2001. p. 139-172.

Smith TW, Glazer K, Ruiz JM, Gallo LC. Hostility, anger, aggressiveness and coronary hearth disease: An interpersonal perspective on personality, emotion, and health. Journal of Personality 2004;72:1217-1270. [PubMed: 15509282]

Spielberger, CD.; Gorsuch, RL.; Lushene, R.; Vagg, PR.; Jacobs, GA. Manual for the State-Trait Anxiety Inventory. Palo Alto, CA: Consulting Psychologists Press; 1983.

Suls J, Bunde J. Anger, anxiety, and depression as risk factors for cardiovascular disease: The problems and implications of overlapping affective dimensions. Psychological Bulletin 2005;131:260-300. [PubMed: 15740422]

Terracciano A, Costa PT. Smoking and the Five-Factor Model of Personality. Addiction 2004;99:472481. [PubMed: 15049747] 
van Loon AJM, Tijhuis M, Surtees PG, Ormel J. Personality and coping: Their relationship with lifestyle risk factors for cancer. Personality and Individual Differences 2001;31:541-553.

Walker MS, Larsen RJ, Zona DM, Govindan R, Fisher EB. Smoking urges and relapse among lung cancer patients: findings from a preliminary retrospective study. Preventative Medicine 2004;39:449-457.

Walker MS, Vidrine DJ, Gritz ER, Larsen RJ, Yan Y, Govidan R, Fisher EB. Smoking relapse during the first year after treatment for early-stage non-small-cell lung cancer. Cancer Epidemiology, Biomarkers, and Prevention 2006;15:2370-2377.

Walker MS, Zona DM, Fisher EB. Depressive symptoms after lung cancer surgery: Their relation to coping style and social support. Psycho-Oncology 2006;15:684-693. [PubMed: 16302291]

Weihs KL, Enright TM, Simmons SJ, Reiss D. Journal of Psychosomatic Research 2000;49:59-68. [PubMed: 11053605]

\section{Acknowledgments}

This work was supported by NIH grant R01 CA8592002 and National Cancer Institute Cancer Center Support grant P30 CA91842 to The Siteman Cancer Center. Data from a subset of this patient sample have been previously presented in Walker, Larsen, Zona, Govindan, Fisher, 2004; Walker, Vidrine, Gritz, Larsen, Yan, Govidan, \& Fisher, 2006; and Walker, Zona, \& Fisher, 2006. 
Table 1

Participant Characteristics

\begin{tabular}{lc}
\hline & Mean (SD) or \% of sample \\
\hline Gender & $50.2 \%$ male \\
Level of education & $50.7 \%$ college or greater \\
Marital status & $70.9 \%$ married or cohabitating \\
Race & $84.2 \%$ Caucasian \\
Income & $46268.49(41222.66)$ \\
Employment status prior to surgery & $56.8 \%$ employed \\
Age started smoking & $16.75(5.38)$ \\
Cigarettes smoked per day prior to surgery & $24.12(12.25)$ \\
\hline
\end{tabular}


Table 2

Partial correlations between age at time of surgery and all other measures

\begin{tabular}{|c|c|c|c|}
\hline & & $\begin{array}{c}\text { Age at time of } \\
\text { surgery (including } \\
\text { smoking covariates) }\end{array}$ & $\begin{array}{c}\text { Age at time of } \\
\text { surgery (including } \\
\text { smoking and gender } \\
\text { covariates) }\end{array}$ \\
\hline Demographics & $\begin{array}{l}\text { Gender } \\
\text { Education } \\
\text { Marital Status } \\
\text { Race } \\
\text { Income } \\
\text { Employment status prior to illness }\end{array}$ & $\begin{array}{l}-.23 * \\
.03 \\
-.03 \\
-.22^{*} \\
-.04 \\
.53\end{array}$ & $\begin{array}{l}- \\
.01 \\
.01 \\
-.26 \\
-.08 \\
.52\end{array}$ \\
\hline BAS/BIS & $\begin{array}{l}\text { BAS Drive } \\
\text { BAS Fun Seeking } \\
\text { BAS Reward Responsiveness } \\
\text { BIS }\end{array}$ & $\begin{array}{r}.09 \\
.12 \\
.07 \\
-.16^{*}\end{array}$ & $\begin{array}{l}.08 \\
.12 \\
.00 \\
-.08\end{array}$ \\
\hline EPQ & $\begin{array}{l}\text { Neuroticism } \\
\text { Extraversion } \\
\text { Psychoticism } \\
\text { Lie }\end{array}$ & $\begin{array}{l}-.20^{*} \\
-.10 \\
-.03 \\
.03\end{array}$ & $\begin{array}{l}-.14^{*} \\
-.09 \\
-.08 \\
.06\end{array}$ \\
\hline BP Aggression & $\begin{array}{l}\text { Anger } \\
\text { Hostility } \\
\text { Physical Aggression } \\
\text { Verbal Aggression } \\
\text { Total Aggression }\end{array}$ & $\begin{array}{l}-.16^{*} \\
-.21 * \\
-.09 \\
-.18^{*} \\
-.18\end{array}$ & $\begin{array}{l}-.20^{*} \\
-.21^{*} \\
-.18 * \\
-.25^{*} \\
-.24^{*}\end{array}$ \\
\hline$\overline{\text { STAI }}$ & Trait Anxiety & $-.14^{*}$ & $-.22^{*}$ \\
\hline$\overline{\mathrm{BDI}}$ & Depression & $-.36^{*}$ & $-.31^{*}$ \\
\hline$\overline{\mathrm{COPE}}$ & $\begin{array}{l}\text { Adaptive Coping } \\
\text { Less Adaptive Coping }\end{array}$ & $\begin{array}{l}-.15^{*} \\
-.26^{*}\end{array}$ & $\begin{array}{l}-.15^{*} \\
-.22 *\end{array}$ \\
\hline
\end{tabular}

Note: $N=203$

${ }^{*} p<.05$

partial correlations; smoking covariates were age participant started smoking and amount participant smoked per day prior to surgery; Gender: $1=$ male, 2=female; Education: 1=high school, 2=at least some college; Marital status: 1=married or cohabitating, 2=not married and no cohabitation; Race: 1=Caucasian, 2=minority; Employment: 1=employed, 2=not employed. 